\title{
Single- and Repeat-dose Oral Toxicity Studies of Lithospermum erythrorhizon Extract in Dogs
}

\author{
Chunja Nam ${ }^{1}$, Jae-Sik Hwang ${ }^{1}$, Myoung-Jun Kim ${ }^{1}$, Young Whan Choi ${ }^{2}$, Kyoung-Goo Han ${ }^{1}$ and Jong-Koo Kang ${ }^{1,3}$ \\ ${ }^{1}$ Biotoxtech Co., Ltd., Cheongju, Korea \\ ${ }^{2}$ Department of Horticultural Bioscience, Pusan National University, Miryang, Korea \\ ${ }^{3}$ Department of Laboratory Animal Medicine, College of Veterinary Medicine, Chungbuk National University, Cheongju, Korea
}

(Received January 9, 2015; Revised March 4, 2015; Accepted March 5, 2015)

\begin{abstract}
Lithospermum erythrorhizon has long been used in traditional Asian medicine for the treatment of diseases, including skin cancer. The oral toxicity of a hexane extract of Lithospermum erythrorhizon root (LEH) was investigated in Beagle dogs by using single escalating doses, two-week dose range-finding, and 4-week oral repeat dosing. In the single dose-escalating oral toxicity study, no animal died, showed adverse clinical signs, or changes in body weight gain at LEH doses of up to $2,000 \mathrm{mg} / \mathrm{kg}$. In a 2 week dose range-finding study, no treatment-related adverse effects were detected by urinalysis, hematology, blood biochemistry, organ weights, or gross and histopathological examinations at doses of up to $500 \mathrm{mg}$ $\mathrm{LEH} / \mathrm{kg} /$ day. In the 4 week repeat-dose toxicity study, a weight loss or decreased weight gain was observed at $300 \mathrm{mg} / \mathrm{kg} /$ day. Although levels of serum triglyceride and total bilirubin were increased in a dose dependent manner, there were no related morphological changes. Based on these findings, the sub-acute no observable adverse effect level for 4-week oral administration of LEH in Beagles was $100 \mathrm{mg} / \mathrm{kg} / \mathrm{day}$.
\end{abstract}

Key words: Lithospermum erythrorhizon extract, Sub-acute toxicity, Beagle dogs

\section{INTRODUCTION}

In traditional Asian medicine, Lithospermum erythrorhizon (LE), Jichi, is used for the treatment of skin rash, pimples, eczema, measles, ulcers, burns, chicken pox, hepatitis, skin cancer, and constipation. LE root extracts have been shown to contain shikonin and its derivatives. These compounds have pharmacological properties that are beneficial for disease treatment, including anti-inflammatory (1), anticancer (2), antimicrobial, anti-allergic (3), and wound healing effects $(4,5)$. Shikonin exerts anti-tumor effects by inhibiting cell growth and inducing apoptosis (6-10). Shikonin, acetylshikonin, and isobutyrylshikonin also produce anti-tumor effects by inhibiting angiogenesis and suppressing tumor growth (11). Shikonin also has anti-tumor activ-

Correspondence to: Jong-Koo Kang, Department of Laboratory Animal Medicine, College of Veterinary Medicine, Chungbuk National University, 1 Chungdae-ro, Seowon-gu, Cheongju, Chungbuk 361763, Korea

E-mail: jkkang@cbu.ac.kr

This is an Open-Access article distributed under the terms of the Creative Commons Attribution Non-Commercial License (http:// creativecommons.org/licenses/by-nc/3.0) which permits unrestricted non-commercial use, distribution, and reproduction in any medium, provided the original work is properly cited. ity via effects on oxidative stress (12).

The main active ingredients of a hexane extract of LE root (LEH; the substance tested in the present study) are naphthoquinones: shikonin, deoxyshikonin, $\beta$-hydroxyisovalerylshikonin, acetylshikonin, and isobutyrylshikonin (3). LEH induced apoptosis in B16F10 cells by activating caspase 3 and inducing sub-G1 cell cycle arrest. It also exhibited anti-cancer activities in vitro and in vivo.

However, there are no published toxicological reports relating to LEH. Therefore, the aim of this study was to evaluate the safety of LEH in dogs by examining its subacute toxicity following repeat oral dosing for 4 weeks.

\section{MATERIALS AND METHODS}

Test substance. The test substance, LEH, was provided by Young Whan Choi at Pusan National University. The active ingredients of LEH are five naphthoquinones (shikonin, deoxyshikonin, $\beta$-hydroxyisovalerylshikonin, acetylshikonin, and isobutyrylshikonin). This LEH (hexane extract) showed in vitro and in vivo anticancer effects of Lithospermum erythrorhizon extract on $\mathrm{B} 16 \mathrm{~F} 10$ murine melanoma (2).

Experimental animals. Male and female Beagle dogs (5 months old) were purchased from Beijing Marshall Bio- 
technology Co., Ltd. (China) and acclimated for 3 weeks prior to drug administration. The animals were housed in a Good Laboratory Practice (GLP) facility with environmentally controlled rooms maintained at a temperature of $21 \pm$ $3^{\circ} \mathrm{C}$, relative humidity of $50 \pm 20 \%$, ventilation $10 \sim 15$ times/ hr, a 12-hr light/dark cycle, and 150 300 lux illumination. Each dog was given $250 \mathrm{~g}$ /day of pellet feed (Cargill Agri Purina Inc.) and sterile tap water was provided ad libitum.

The ethical committee at Biotoxtech Co., Ltd. approved the use of the animals and the study design. The studies were conducted in accordance with the GLP Regulation and Test Guideline of Standards for Toxicity Studies of Drugs, issued by the Korean Food and Drug Administration.

Dose-escalating toxicity study (DES) of LEH. In brief, 2 dogs or 1 dog in each group were weighed and orally administered either LEH with dose escalation at four-day intervals $(500 \rightarrow 1,000 \rightarrow 2,000 \mathrm{mg} / \mathrm{kg}$ ) or its vehicle (corn oil) at a volume of $5 \mathrm{~mL} / \mathrm{kg}$. Clinical signs and mortality were examined every day until Day 14 after the final dose was administered; body weights were recorded on Days 0 , $1,3,4,5,7,8,9,11,15$, and 21 .

Two-week repeated oral dose range-finding study. Two animals of both sexes in each group were weighed and administered with either LEH at doses of 100, 300, and 500 $\mathrm{mg} / \mathrm{kg} /$ day or its vehicle at a volume of $2 \mathrm{ml} / \mathrm{kg}$ for 2 weeks. Clinical signs and mortality were examined every day. Body weights and feed consumption were measured on Day 0 and then twice a week and once a week, respectively. Urinalysis, hematology, and blood biochemistry were examined prior to the first dose and on Day 14 of the dosing period. All dogs were sacrificed under deep anesthesia with a thiopental sodium overdose. Complete gross postmortem and histopathological examinations were conducted.

Four-week repeated dose oral toxicity study with a two-week recovery and toxicokinetic analysis. Three animals of both sexes in each group were weighed and administered with either LEH at doses of 30,100 , and $300 \mathrm{mg} / \mathrm{kg}$ / day or its vehicle at a volume of $2 \mathrm{~mL} / \mathrm{kg}$ for 4 weeks. This study also assessed the reversibility of any adverse findings during a two-week recovery period and determined systemic exposure to the test substance in a toxicokinetic study. Clinical signs and mortality were examined every day, and body weights and feed consumption were measured on Day 0 and once a week during the dosing and recovery periods. Ophthalmologic examination, electrocardiography (ECG), urinalysis, hematology, and blood chemistry were examined prior to dosing, in Week 4 of the dosing period, and in Week 2 of the recovery period. At the end of the dosing and recovery periods, dogs were sacrificed under deep anesthesia with a thiopental sodium overdose. Complete gross postmortem and histopathological examinations were conducted.
For the ophthalmologic examination, a mydriatic agent (SOPTO-AtROPINE®, Alcon-Couvreur, Belgium) was instilled into both eyes and examination of the anterior segment, transparent media, and ocular fundus was carried out macroscopically using an ophthalmoscope (PRACTITIONER, Keeler, UK).

The heart rate, R-R interval, P-R interval, QRS duration, Q-T interval, QT correcting, and mean electric axis (AXIS) were recorded using an ECG system (Cardiomax FX-3010, FUKUDA DENSHI, Japan).

For urinalysis, fresh urine samples were collected within $3 \mathrm{hr}$ and analyzed for $\mathrm{pH}$, protein, glucose, ketone bodies, bilirubin, red blood cells (RBC), color, and turbidity using either an autoanalyzer (cobas u 411, Roche, Germany) or test kit (Combur10Test ${ }^{\circledR} \mathrm{M}$ stick; Roche Diagnostics, Mannheim, Germany).

For hematology, $1 \mathrm{~mL}$ of whole blood was collected into a CBC bottle containing EDTA and examined using a hematological autoanalyzer (ADVIA 2120i, SIEMENS, Germany). The hematological parameters examined included the total RBC count, hemoglobin concentration (HGB), hematocrit $(\mathrm{HCT})$, mean cell volume (MCV), mean cell hemoglobin $(\mathrm{MCH})$, mean cell hemoglobin concentration (MCHC), the platelet count (PLT), the total white blood cell count (WBC), WBC differential counting (neutrophil, lymphocyte, monocyte, eosinophil, and basophil ratio), reticulocyte (Reti), prothrombin time (PT), and activated partial thromboplastin time (APTT). For the blood coagulation time test, plasma samples were examined using an automatic analyzer (Coapresta 2000, SEKISUI, Japan) for PT and APTT.

For blood biochemistry analyses, $3 \mathrm{~mL}$ blood was centrifuged to collect serum samples. Serum samples were analyzed using an automatic analyzer (7080, HITACHI, Japan). The parameters examined included alanine aminotransferase (ALT), aspartate aminotransferase (AST), alkaline phosphatase (ALP), $\gamma$-glutamyl transpeptidase (GGT), blood urea nitrogen (BUN), creatinine (Crea), total bilirubin (TBili), albumin (Alb), globulin ( $\alpha 1, \alpha 2, \beta, \gamma), \mathrm{A} / \mathrm{G}$ ratio, total cholesterol (T-Chol), triglycerides (TG), glucose (Glu), phosphorus $(\mathrm{P})$, calcium $(\mathrm{Ca})$, potassium $(\mathrm{K})$, and chloride (Cl).

All surviving animals were exsanguinated under thiopental sodium anesthesia. All animals were subjected to complete gross postmortem and histopathological examinations.

Absolute and relative organ weights were determined for the brain, pituitary gland, thymus, thyroid gland, heart, kidney, liver, adrenal, and spleen, in addition to the testis, prostate, and epididymis, or uterus and ovary, as appropriate.

The following organs were excised and fixed in $10 \%$ neutral buffered formalin: brain, pituitary, thyroid and parathyroid, thymus, lung, trachea, heart, liver, gall bladder, spleen, kidney, adrenal, kidney, lymph nodes (submandibular and 
mesenteric), submandibular gland, esophagus, stomach, duodenum, jejunum, ileum, cecum, colon, rectum, pancreas, urinary bladder, mammary gland, skin, tongue, thoracic spinal cord, skeletal muscle, sciatic nerve, eye with optic nerve, and femur with bone marrow, as well as the testis, epididymis, and prostate or uterus, ovary, and vagina, as appropriate. The eyes and testis were fixed in Davidson's solution. Decalcification was performed with Calci-ClearRapid $^{\mathrm{TM}}$ solution (National Diagnostics, USA).

After fixing, the organs and tissues from all study groups were prepared for microscopic examination by routine tissue processing, embedding in paraffin, sectioning, and staining with hematoxylin and eosin.

For toxicokinetic analyses, blood samples were collected on Days 1 and 28 as follows at 0, 0.25, 0.75, 1, 2, 4, 6, 10, and $24 \mathrm{hr}$ after administration (10 time-points). Blood samples were collected from the cephalic vein using heparinized syringes and centrifuged at $12,000 \mathrm{rpm}$ for $3 \mathrm{~min}$ at $4^{\circ} \mathrm{C}$, and plasma samples were stored at -80 to $-60^{\circ} \mathrm{C}$. Analyses of plasma levels of the test substance in plasma were performed using a liquid chromatography-tandem mass spectrometry (LC/MS/MS) method (Prominence, SHIMADZU Co., Ltd., Japan and API5500, AB Sciex, Pte. Ltd., USA). The following parameters were calculated for acetylshikonin, as an index analyte; AUC (area under the plasma concentration-time curve), $\mathrm{C}_{\max }$ (maximum observed plasma concentration), $\mathrm{T}_{\max }$ (time of occurrence of $\mathrm{C}_{\max }$ ), and $t_{1 / 2}$ (apparent terminal half-life).

Statistical analyses were carried out using the SAS program (version 9.1.3, SAS Institute Inc.; Cary, NC, USA). The body weight, food consumption, ECG, clinical pathology data, and organ weights from the control and test substance-treated groups were analyzed by one-way of variance (ANOVA) following Bartlett's test to determine the homogeneity of variance $(p<0.05$, two-sided). If variances were not homogeneous, the data were analyzed using the nonparametric Kruskal-Wallis test. If statistical significance was observed $(p<0.05)$, the control and treated groups were compared using either Dunnett's multiple comparison test (homogeneous data, $p<0.05$, two-sided) or Steel's multiple comparison test (heterogeneous data, $p<0.05$, twosided).

\section{RESULTS AND DISCUSSION}

Single oral DES. Throughout the experimental period, no dead or moribund animals of either sex were observed in the control and treatment groups. Diarrhea and/or vomiting were observed after oral administration of 500, 1,000, and $2,000 \mathrm{mg} / \mathrm{kg} \mathrm{LEH}$. There were no significant differences in body weight gain (Fig. 1) or necropsy findings in the control and treatment groups. Therefore, the maximum tolerance dose (MTD) of LEH was $2,000 \mathrm{mg} / \mathrm{kg}$ under the conditions employed in this study.
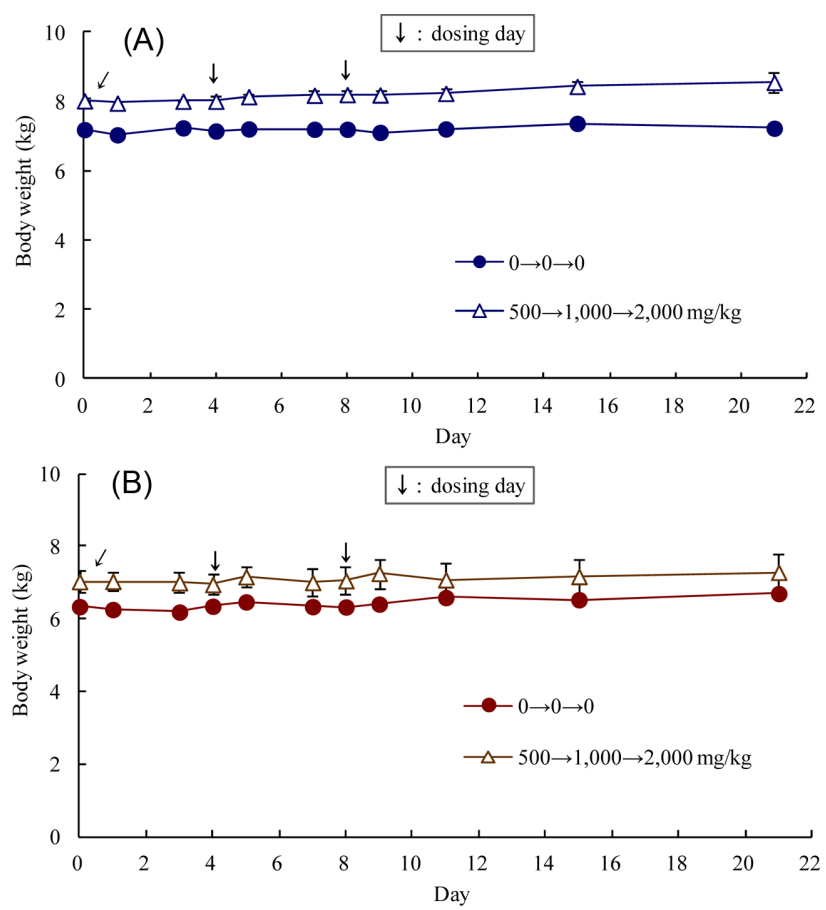

Fig. 1. Body weights of male (A) and female (B) Beagle dogs during dose escalation of Lithospermum erythrorhizon hexane extract.

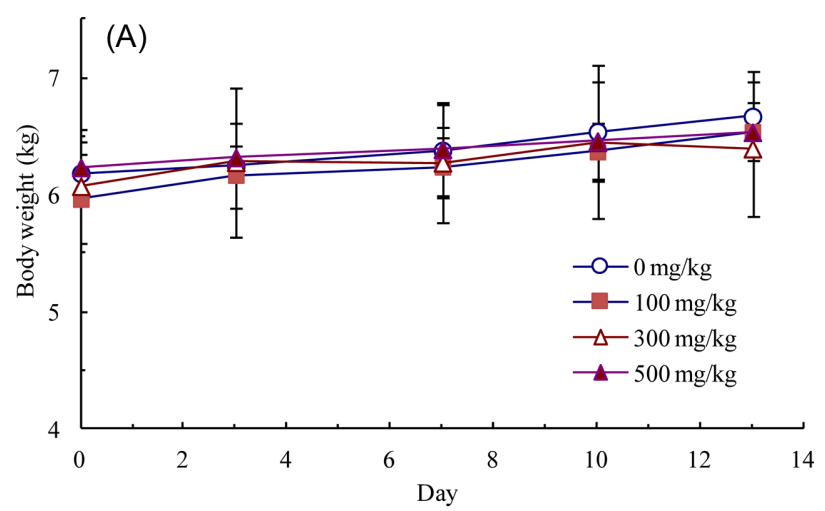

(B)

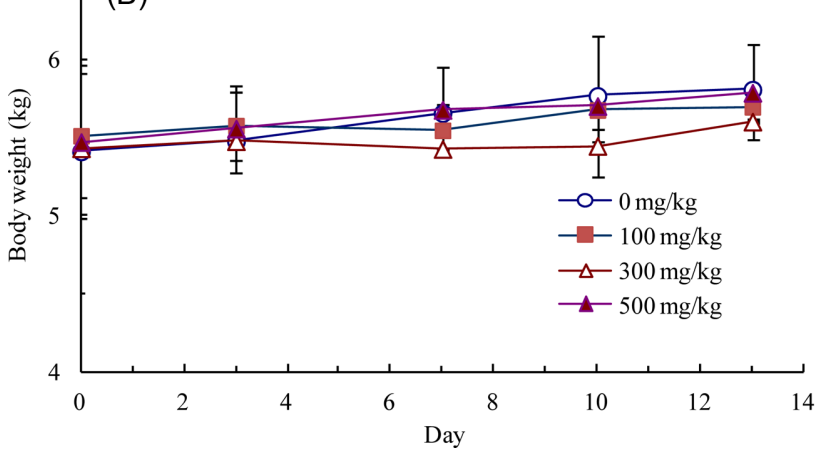

Fig. 2. Body weights of male (A) and female (B) Beagle dogs during the 2-week Lithospermum erythrorhizon hexane extract dose range-finding study. Each data point is presented as mean \pm standard deviation. 
Two-week repeated oral dose range-finding study. There were no dead or moribund animals in the control or treatment groups. Compound-colored stools, soft stools, and/or diarrhea were often observed in all of the LEH-treated groups, and vomiting was sporadically observed. At 500 $\mathrm{mg} / \mathrm{kg} /$ day, salivation was often observed. There were no significant differences in body weight gain (Fig. 2) and food consumption between the study groups. T-Bili was dosedependently increased. No treatment-related adverse effects on urinalysis, hematology, organ weights, or gross and histopathological examinations were detected. Therefore, 300 $\mathrm{mg} / \mathrm{kg} /$ day was selected as a high dose for the 4 week repeatdose oral toxicity study. Dose levels of 100 and $30 \mathrm{mg} / \mathrm{kg}$ / day were also selected as mid and low doses, respectively.

Four-week repeated dose oral toxicity study with a two-week recovery and toxicokinetic analysis. During the dosing period, there were dose-dependent increases in salivation, vomiting, soft stools and/or diarrhea in LEHtreated groups. These symptoms were not observed during the recovery period, after dosing had ceased. In Week 4, body weight loss $(-6 \%)$ or a decrease in body weight gain $(>50 \%)$ was observed in males and females, respectively, receiving $300 \mathrm{mg} \mathrm{LEH} / \mathrm{kg} / \mathrm{day}$, as compared to the control animals (Fig. 3). The weight loss or reduced weight gain was related to vomiting and diarrhea, and were thus test substance-related effects. No significant differences in food
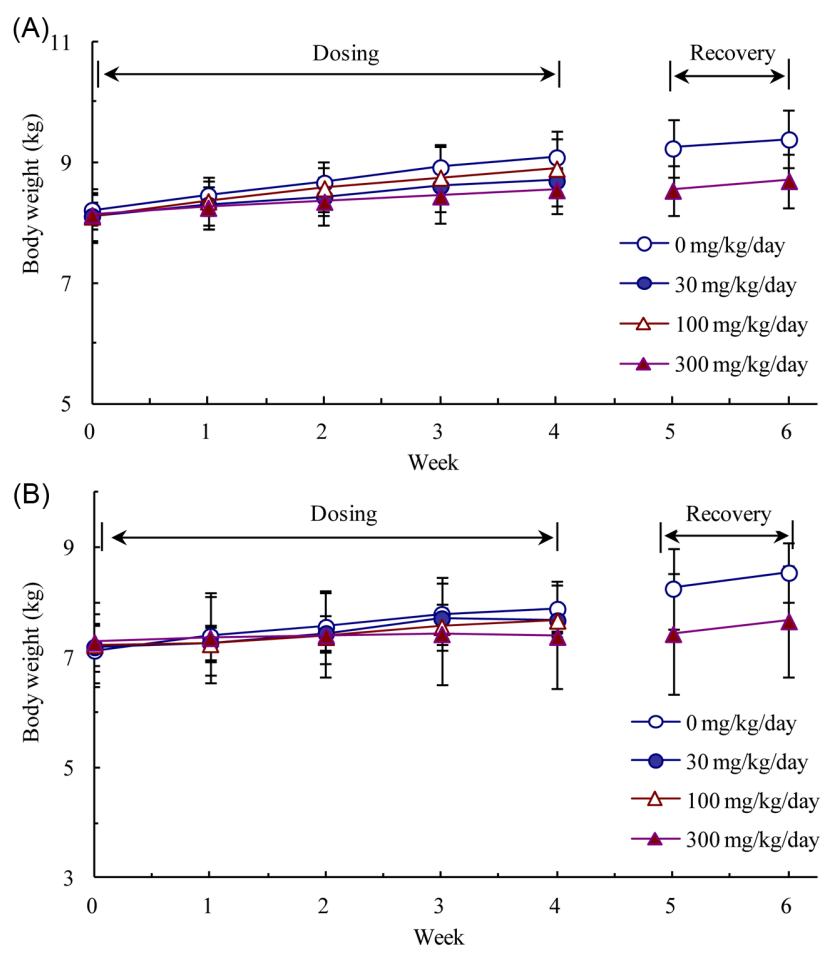

Fig. 3. Body weights of male (A) and female (B) Beagle dogs during 4-week administration of the indicated doses of Lithospermum erythrorhizon hexane extract and a 2-week recovery period. Each data point is presented as mean \pm standard deviation.

Table 1. Electrocardiogram of dogs administered with Lithospermum erythrorhizon hexane extract or its vehicle for 4 weeks

\begin{tabular}{|c|c|c|c|c|c|}
\hline & & \multicolumn{4}{|c|}{ Dose (mg/kg/day) } \\
\hline & & Control & 30 & 100 & 300 \\
\hline \multicolumn{6}{|l|}{ Males } \\
\hline \multirow{7}{*}{ Week 0 (Pre) } & HR (bpm) & $118 \pm 11$ & $109 \pm 11$ & $104 \pm 14$ & $113 \pm 14$ \\
\hline & R-R interval (sec) & $0.511 \pm 0.050$ & $0.550 \pm 0.054$ & $0.579 \pm 0.074$ & $0.533 \pm 0.068$ \\
\hline & P-R interval (sec) & $0.097 \pm 0.008$ & $0.101 \pm 0.006$ & $0.099 \pm 0.005$ & $0.093 \pm 0.012$ \\
\hline & QRS duration (sec) & $0.053 \pm 0.006$ & $0.051 \pm 0.006$ & $0.057 \pm 0.006$ & $0.048 \pm 0.002$ \\
\hline & QT interval (sec) & $0.195 \pm 0.003$ & $0.199 \pm 0.006$ & $0.207 \pm 0.015$ & $0.196 \pm 0.006$ \\
\hline & QT correcting & $0.273 \pm 0.011$ & $0.268 \pm 0.006$ & $0.271 \pm 0.007$ & $0.269 \pm 0.010$ \\
\hline & Axis (degree) & $68 \pm 11$ & $72 \pm 7$ & $73 \pm 1$ & $69 \pm 7$ \\
\hline \multirow{7}{*}{ Week 4} & HR (bpm) & $124 \pm 6$ & $105 \pm 15$ & $116 \pm 13$ & $122 \pm 7$ \\
\hline & R-R interval (sec) & $0.485 \pm 0.023$ & $0.572 \pm 0.079$ & $0.521 \pm 0.055$ & $0.493 \pm 0.030$ \\
\hline & P-R interval (sec) & $0.098 \pm 0.009$ & $0.105 \pm 0.007$ & $0.101 \pm 0.001$ & $0.095 \pm 0.011$ \\
\hline & QRS duration (sec) & $0.049 \pm 0.004$ & $0.051 \pm 0.002$ & $0.050 \pm 0.004$ & $0.047 \pm 0.002$ \\
\hline & QT interval (sec) & $0.194 \pm 0.004$ & $0.203 \pm 0.008^{*}$ & $0.196 \pm 0.004$ & $0.196 \pm 0.000$ \\
\hline & QT correcting & $0.278 \pm 0.002$ & $0.268 \pm 0.009$ & $0.272 \pm 0.010$ & $0.279 \pm 0.008$ \\
\hline & Axis (degree) & $69 \pm 8$ & $61 \pm 15$ & $69 \pm 6$ & $74 \pm 3$ \\
\hline \multirow{7}{*}{ Week 2 of Recovery } & HR (bpm) & $99 \pm 8$ & & & $105 \pm 15$ \\
\hline & R-R interval (sec) & $0.605 \pm 0.051$ & & & $0.577 \pm 0.079$ \\
\hline & P-R interval (sec) & $0.105 \pm 0.001$ & & & $0.097 \pm 0.011$ \\
\hline & QRS duration (sec) & $0.050 \pm 0.007$ & & & $0.055 \pm 0.004$ \\
\hline & QT interval (sec) & $0.206 \pm 0.008$ & & & $0.198 \pm 0.003$ \\
\hline & QT correcting & $0.265 \pm 0.001$ & & & $0.261 \pm 0.014$ \\
\hline & Axis (degree) & $67 \pm 3$ & & & $67 \pm 7$ \\
\hline
\end{tabular}


Table 1. Continued

\begin{tabular}{|c|c|c|c|c|c|}
\hline & & \multicolumn{4}{|c|}{ Dose (mg/kg/day) } \\
\hline & & Control & 30 & 100 & 300 \\
\hline \multicolumn{6}{|l|}{ Females } \\
\hline \multirow{7}{*}{ Week 0 (Pre) } & HR (bpm) & $110 \pm 11$ & $116 \pm 12$ & $109 \pm 23$ & $107 \pm 15$ \\
\hline & R-R interval (sec) & $0.549 \pm 0.053$ & $0.520 \pm 0.051$ & $0.565 \pm 0.121$ & $0.564 \pm 0.079$ \\
\hline & P-R interval (sec) & $0.106 \pm 0.004$ & $0.106 \pm 0.014$ & $0.102 \pm 0.011$ & $0.109 \pm 0.008$ \\
\hline & QRS duration (sec) & $0.051 \pm 0.005$ & $0.056 \pm 0.004$ & $0.055 \pm 0.004$ & $0.051 \pm 0.006$ \\
\hline & QT interval (sec) & $0.197 \pm 0.003$ & $0.196 \pm 0.004$ & $0.199 \pm 0.012$ & $0.202 \pm 0.009$ \\
\hline & QT correcting & $0.266 \pm 0.010$ & $0.272 \pm 0.009$ & $0.266 \pm 0.012$ & $0.269 \pm 0.009$ \\
\hline & Axis (degree) & $68 \pm 7$ & $66 \pm 4$ & $74 \pm 4$ & $72 \pm 7$ \\
\hline \multirow{7}{*}{ Week 4} & HR (bpm) & $117 \pm 14$ & $123 \pm 8$ & $128 \pm 5$ & $108 \pm 17$ \\
\hline & R-R interval (sec) & $0.515 \pm 0.060$ & $0.489 \pm 0.031$ & $0.467 \pm 0.016$ & $0.564 \pm 0.081$ \\
\hline & P-R interval (sec) & $0.108 \pm 0.006$ & $0.106 \pm 0.007$ & $0.101 \pm 0.012$ & $0.105 \pm 0.010$ \\
\hline & QRS duration (sec) & $0.053 \pm 0.005$ & $0.054 \pm 0.005$ & $0.058 \pm 0.002$ & $0.054 \pm 0.005$ \\
\hline & QT interval (sec) & $0.196 \pm 0.005$ & $0.193 \pm 0.005$ & $0.193 \pm 0.005$ & $0.202 \pm 0.009$ \\
\hline & QT correcting & $0.274 \pm 0.010$ & $0.276 \pm 0.006$ & $0.283 \pm 0.003$ & $0.269 \pm 0.009$ \\
\hline & Axis (degree) & $68 \pm 3$ & $68 \pm 8$ & $74 \pm 10$ & $73 \pm 5$ \\
\hline \multirow{7}{*}{ Week 2 of Recovery } & HR (bpm) & $92 \pm 3$ & & & $100 \pm 7$ \\
\hline & R-R interval (sec) & $0.649 \pm 0.018$ & & & $0.601 \pm 0.042$ \\
\hline & P-R interval (sec) & $0.124 \pm 0.012$ & & & $0.104 \pm 0.014$ \\
\hline & QRS duration (sec) & $0.054 \pm 0.006$ & & & $0.050 \pm 0.003$ \\
\hline & QT interval (sec) & $0.206 \pm 0.008$ & & & $0.200 \pm 0.000$ \\
\hline & QT correcting & $0.255 \pm 0.007$ & & & $0.258 \pm 0.009$ \\
\hline & Axis (degree) & $66 \pm 4$ & & & $71 \pm 4$ \\
\hline
\end{tabular}

Significantly different from the control by Dunnett's t-test: * $p<0.05$.

Table 2. Urinalysis data of Beagle dogs orally administered with Lithospermum erythrorhizon hexane extract for 4 weeks

\begin{tabular}{|c|c|c|c|c|c|c|c|c|c|c|c|c|c|c|c|c|c|c|c|c|c|}
\hline \multirow{3}{*}{\multicolumn{2}{|c|}{$\begin{array}{l}\text { Sex } \\
\text { Dose }(\mathrm{mg} / \mathrm{kg} / \text { day }) \\
\end{array}$}} & \multicolumn{8}{|c|}{ Week 0 (Pre) } & \multicolumn{8}{|c|}{ Week 4 of Dosing } & \multicolumn{4}{|c|}{ Week 2 of Recovery } \\
\hline & & \multicolumn{4}{|c|}{ Male } & \multicolumn{4}{|c|}{ Female } & \multicolumn{4}{|c|}{ Male } & \multicolumn{4}{|c|}{ Female } & \multicolumn{2}{|c|}{ Male } & \multicolumn{2}{|c|}{ Female } \\
\hline & & 0 & 30 & 100 & 300 & 0 & 30 & 100 & 300 & 0 & 30 & 100 & 300 & 0 & 30 & 100 & 300 & 0 & 300 & 0 & 300 \\
\hline No. of animals & & 5 & 3 & 3 & 5 & 5 & 3 & 3 & 5 & 5 & 3 & 3 & 5 & 5 & 3 & 3 & 5 & 2 & 2 & 2 & 2 \\
\hline \multirow[t]{4}{*}{ Color } & Pale yellow & 1 & 3 & & 2 & 4 & 2 & 3 & 2 & 2 & 2 & & & 1 & & 1 & & & & & \\
\hline & Yellow & 4 & & 3 & 3 & 1 & 1 & & 3 & 3 & & 3 & 1 & 4 & 2 & 2 & 1 & 2 & 2 & 2 & 2 \\
\hline & Amber & & & & & & & & & & 1 & & 4 & & 1 & & 1 & & & & \\
\hline & Brown & & & & & & & & & & & & & & & & 3 & & & & \\
\hline \multirow[t]{6}{*}{$\mathrm{pH}$} & 5 & & & & & & & & & & & & & & 2 & & 1 & & & 1 & \\
\hline & 6 & & & & 1 & & & & & & & & & & & & & 2 & & 1 & \\
\hline & 6.5 & 1 & & & & & & & & & & & & & & 1 & 1 & & & & 1 \\
\hline & 7 & & & & 1 & & & & & & & & & & 1 & 1 & & & & & \\
\hline & 8 & 3 & 2 & 1 & 3 & 2 & 3 & 3 & 3 & 5 & 3 & 3 & 4 & 4 & & 1 & 3 & & 2 & & 1 \\
\hline & 9 & 1 & 1 & & 2 & 3 & & & 2 & & & & 1 & 1 & & & & & & & \\
\hline \multirow{2}{*}{$\begin{array}{l}\text { Protein } \\
(\mathrm{mg} / \mathrm{dL})\end{array}$} & - & 3 & 3 & 2 & 5 & 4 & 3 & 3 & 5 & 3 & 2 & 2 & 2 & 5 & 2 & 3 & 1 & 1 & 1 & 2 & 2 \\
\hline & 25 & 2 & & 1 & & 1 & & & & 2 & 1 & 1 & 3 & & 1 & & 4 & 1 & 1 & & \\
\hline $\begin{array}{l}\text { Glucose } \\
\text { (mg/dL) }\end{array}$ & $\begin{array}{l}5 \\
50\end{array}$ & 5 & 3 & 3 & 5 & 5 & 3 & 3 & 5 & 5 & 3 & 3 & 5 & 5 & 3 & 3 & 5 & 2 & 2 & 2 & 2 \\
\hline \multirow{2}{*}{$\begin{array}{l}\text { Ketone body } \\
(\mathrm{mg} / \mathrm{dL})\end{array}$} & - & 5 & 3 & 2 & 5 & 5 & 3 & 3 & 5 & 5 & 3 & 2 & 2 & 4 & 2 & 2 & 2 & 2 & 2 & 1 & 2 \\
\hline & 5 & & & 1 & & & & & & & & 1 & 3 & 1 & 1 & 1 & 3 & & & 1 & \\
\hline \multirow{3}{*}{$\begin{array}{l}\text { Bilirubin } \\
(\mathrm{mg} / \mathrm{dL})\end{array}$} & - & 5 & 3 & 2 & 5 & 5 & 3 & 3 & 5 & 5 & 3 & 1 & 5 & 5 & 2 & 3 & 3 & 2 & 2 & 2 & 2 \\
\hline & 1 & & & 1 & & & & & & & & 1 & & & 1 & & 2 & & & & \\
\hline & 3 & & & & & & & & & & & 1 & & & & & & & & & \\
\hline
\end{tabular}


Table 2. Continued

\begin{tabular}{|c|c|c|c|c|c|c|c|c|c|c|c|c|c|c|c|c|c|c|c|c|c|}
\hline \multirow{3}{*}{$\frac{\text { Sex }}{\text { Dose }(\mathrm{mg} / \mathrm{kg} / \text { day })}$} & & \multicolumn{8}{|c|}{ Week 0 (Pre) } & \multicolumn{8}{|c|}{ Week 4 of Dosing } & \multicolumn{4}{|c|}{ Week 2 of Recovery } \\
\hline & & \multicolumn{4}{|c|}{ Male } & \multicolumn{4}{|c|}{ Female } & \multicolumn{4}{|c|}{ Male } & \multicolumn{4}{|c|}{ Female } & \multicolumn{2}{|c|}{ Male } & \multicolumn{2}{|c|}{ Female } \\
\hline & & 0 & 30 & 100 & 300 & 0 & 30 & 100 & 300 & 0 & 30 & 100 & 300 & 0 & 30 & 100 & 300 & 0 & 300 & 0 & 300 \\
\hline Erythrocyte & - & 2 & 1 & 1 & 5 & 4 & 3 & 3 & 5 & 3 & 1 & 2 & 4 & 5 & 3 & 3 & 4 & 1 & 2 & 2 & 2 \\
\hline$($ Ery $/ \mu \mathrm{L})$ & 10 & 2 & 2 & 2 & & & & & & 1 & 1 & & & & & & & 1 & & & \\
\hline & 25 & 1 & & & & & & & & 1 & 1 & 1 & & & & & 1 & & & & \\
\hline & 50 & & & & & 1 & & & & & & & & & & & & & & & \\
\hline & 150 & & & & & & & & & & & & 1 & & & & & & & & \\
\hline Cast $^{\dagger}$ & $\begin{array}{c}0 \\
1 \sim 5\end{array}$ & 5 & 3 & 3 & 5 & 5 & 3 & 3 & 5 & 5 & 3 & 3 & 5 & 5 & 3 & 3 & 5 & 2 & 2 & 2 & 2 \\
\hline Epithelia cell $^{\dagger}$ & $\begin{array}{c}0 \\
1 \sim 5\end{array}$ & 5 & 3 & 3 & 5 & 5 & 3 & 3 & 5 & 5 & 3 & 3 & 5 & 5 & 3 & 3 & 5 & 2 & 2 & 2 & 2 \\
\hline
\end{tabular}

${ }^{\dagger}$ : Number of counts in 20 visual fields, $400 \times$.

consumption were observed during the dosing and recovery periods (data not shown).

In addition, there were no considerable changes in ophthalmologic parameters (data not shown). In Week 4, the ECG exhibited a significant increase in the QT interval in males receiving $30 \mathrm{mg} \mathrm{LEH} / \mathrm{kg} /$ day $(p<0.05)$ (Table 1$)$. The increase in QT interval in males at $30 \mathrm{mg} / \mathrm{kg} /$ day was not considered to be a significant difference, as compared to pre-dosing values. In the recovery group, there were no abnormal changes in ECG.

Table 3. Urine specific gravity

\begin{tabular}{lcccc}
\hline \hline Sex & Dose $(\mathrm{mg} / \mathrm{kg} /$ day) & Pre & Week 4 & Week 2 (recovery) \\
\hline Males & 0 & $1.020 \pm 0.008$ & $1.026 \pm 0.018$ & $1.017 \pm 0.007$ \\
& 30 & $1.012 \pm 0.007$ & $1.022 \pm 0.013$ & \\
& 100 & $1.027 \pm 0.007$ & $1.028 \pm 0.008$ & $1.024 \pm 0.021$ \\
\hline Females & $1.020 \pm 0.008$ & $1.038 \pm 0.006$ & $1.029 \pm 0.013$ \\
& 0 & $1.025 \pm 0.010$ & $1.037 \pm 0.006$ & $1.016 \pm 0.004$ \\
\hline
\end{tabular}

Table 4. Hematological values of beagle dogs at week 0 (pre-dosing)

\begin{tabular}{|c|c|c|c|c|c|}
\hline \multicolumn{2}{|c|}{ Dose (mg/kg/day) } & Control & 30 & 100 & 300 \\
\hline \multicolumn{6}{|l|}{ Males } \\
\hline \multicolumn{2}{|c|}{$\mathrm{RBC}\left(\times 10^{6}\right.$ cells $\left./ \mu \mathrm{L}\right)$} & $6.28 \pm 0.52$ & $6.79 \pm 0.78$ & $6.80 \pm 0.51$ & $6.72 \pm 0.57$ \\
\hline \multicolumn{2}{|l|}{ HGB $(g / d L)$} & $14.6 \pm 1.1$ & $15.3 \pm 1.3$ & $15.3 \pm 0.8$ & $15.5 \pm 1.2$ \\
\hline \multicolumn{2}{|l|}{$\operatorname{HCT}(\%)$} & $40.6 \pm 3.2$ & $41.9 \pm 3.2$ & $42.2 \pm 2.4$ & $43.0 \pm 3.0$ \\
\hline \multicolumn{2}{|l|}{ Reti (\%) } & $1.23 \pm 0.31$ & $1.22 \pm 0.34$ & $0.92 \pm 0.26$ & $1.42 \pm 0.45$ \\
\hline \multirow[t]{3}{*}{$\mathrm{RBC}$ index } & MCV (fL) & $64.6 \pm 1.3$ & $61.9 \pm 2.5$ & $62.1 \pm 1.3$ & $64.2 \pm 2.2$ \\
\hline & $\mathrm{MCH}(\mathrm{pg})$ & $23.3 \pm 0.6$ & $22.7 \pm 0.8$ & $22.5 \pm 0.7$ & $23.1 \pm 0.8$ \\
\hline & $\mathrm{MCHC}(\mathrm{g} / \mathrm{dL})$ & $36.0 \pm 0.5$ & $36.6 \pm 0.4$ & $36.2 \pm 0.4$ & $36.1 \pm 0.4$ \\
\hline \multicolumn{2}{|c|}{$\operatorname{PLT}\left(\times 10^{3}\right.$ cells $\left./ \mu \mathrm{L}\right)$} & $232 \pm 56$ & $248 \pm 33$ & $286 \pm 27$ & $293 \pm 77$ \\
\hline \multicolumn{2}{|c|}{$\mathrm{WBC}\left(\times 10^{3}\right.$ cells $\left./ \mu \mathrm{L}\right)$} & $8.99 \pm 1.87$ & $10.33 \pm 2.26$ & $9.05 \pm 1.10$ & $9.74 \pm 0.91$ \\
\hline WBC & NEU & $50.6 \pm 6.8$ & $44.1 \pm 4.5$ & $49.2 \pm 3.1$ & $53.4 \pm 6.6$ \\
\hline & LYM & $35.1 \pm 7.1$ & $39.8 \pm 3.4$ & $35.6 \pm 3.9$ & $35.2 \pm 5.1$ \\
\hline \multirow{3}{*}{ counting $(\%)$} & MONO & $5.8 \pm 1.0$ & $6.8 \pm 2.2$ & $5.9 \pm 2.4$ & $5.2 \pm 1.4$ \\
\hline & EOS & $7.5 \pm 2.1$ & $8.2 \pm 1.3$ & $8.0 \pm 3.8$ & $5.0 \pm 2.7$ \\
\hline & BASO & $0.5 \pm 0.2$ & $0.7 \pm 0.2$ & $0.8 \pm 0.1$ & $0.7 \pm 0.5$ \\
\hline PT (sec) & & $9.7 \pm 0.3$ & $9.5 \pm 0.1$ & $9.6 \pm 0.1$ & $9.7 \pm 0.3$ \\
\hline APTT (sec) & & $14.1 \pm 1.0$ & $15.6 \pm 0.3$ & $15.3 \pm 0.7$ & $15.1 \pm 0.6$ \\
\hline
\end{tabular}


Table 4. Continued

\begin{tabular}{|c|c|c|c|c|c|}
\hline \multicolumn{2}{|c|}{ Dose (mg/kg/day) } & Control & 30 & 100 & 300 \\
\hline \multicolumn{6}{|l|}{ Females } \\
\hline \multicolumn{2}{|c|}{$\mathrm{RBC}\left(\times 10^{6}\right.$ cells $\left./ \mu \mathrm{L}\right)$} & $6.55 \pm 0.37$ & $7.00 \pm 0.68$ & $6.32 \pm 0.28$ & $6.14 \pm 0.56$ \\
\hline \multicolumn{2}{|l|}{$\mathrm{HGB}(\mathrm{g} / \mathrm{dL})$} & $15.0 \pm 0.9$ & $15.9 \pm 1.6$ & $14.2 \pm 0.7$ & $14.5 \pm 1.8$ \\
\hline \multicolumn{2}{|l|}{ HCT (\%) } & $41.1 \pm 1.9$ & $43.2 \pm 4.1$ & $39.4 \pm 1.8$ & $39.4 \pm 3.9$ \\
\hline \multicolumn{2}{|l|}{ Reti (\%) } & $1.29 \pm 0.37$ & $0.94 \pm 0.15$ & $1.10 \pm 0.39$ & $0.80 \pm 0.26$ \\
\hline \multirow[t]{3}{*}{$\mathrm{RBC}$ index } & MCV (fL) & $62.8 \pm 2.2$ & $61.7 \pm 0.4$ & $62.4 \pm 2.7$ & $64.2 \pm 2.8$ \\
\hline & $\mathrm{MCH}(\mathrm{pg})$ & $23.0 \pm 0.9$ & $22.7 \pm 0.4$ & $22.5 \pm 0.7$ & $23.6 \pm 1.3$ \\
\hline & $\mathrm{MCHC}(\mathrm{g} / \mathrm{dL})$ & $36.6 \pm 0.6$ & $36.7 \pm 0.5$ & $36.0 \pm 0.6$ & $36.8 \pm 0.8$ \\
\hline \multicolumn{2}{|c|}{$\operatorname{PLT}\left(\times 10^{3}\right.$ cells $\left./ \mu \mathrm{L}\right)$} & $253 \pm 35$ & $301 \pm 17$ & $262 \pm 12$ & $283 \pm 42$ \\
\hline \multicolumn{2}{|c|}{$\mathrm{WBC}\left(\times 10^{3}\right.$ cells $\left./ \mu \mathrm{L}\right)$} & $8.45 \pm 1.58$ & $7.69 \pm 2.41$ & $9.37 \pm 2.75$ & $7.63 \pm 1.77$ \\
\hline WBC & NEU & $53.2 \pm 4.7$ & $53.1 \pm 5.5$ & $57.8 \pm 4.8$ & $52.9 \pm 4.4$ \\
\hline differential & LYM & $35.9 \pm 5.1$ & $36.8 \pm 3.9$ & $31.4 \pm 6.1$ & $36.5 \pm 4.7$ \\
\hline \multirow[t]{3}{*}{ counting $(\%)$} & MONO & $5.5 \pm 0.6$ & $5.6 \pm 0.5$ & $6.8 \pm 1.6$ & $5.5 \pm 0.8$ \\
\hline & EOS & $4.6 \pm 3.3$ & $3.5 \pm 2.0$ & $3.4 \pm 0.9$ & $4.0 \pm 1.5$ \\
\hline & BASO & $0.6 \pm 0.4$ & $0.6 \pm 0.5$ & $0.3 \pm 0.1$ & $0.8 \pm 0.4$ \\
\hline PT (sec) & & $10.2 \pm 1.3$ & $9.6 \pm 0.0$ & $9.9 \pm 0.4$ & $10.0 \pm 0.7$ \\
\hline APTT (sec) & & $15.8 \pm 1.9$ & $15.2 \pm 1.9$ & $15.1 \pm 1.6$ & $15.7 \pm 2.4$ \\
\hline
\end{tabular}

Table 5. Hematological values of Beagle dogs administered with Lithospermum erythrorhizon hexane extract or its vehicle

\begin{tabular}{|c|c|c|c|c|c|c|c|}
\hline \multirow{2}{*}{\multicolumn{2}{|c|}{ Dose (mg/kg/day) }} & \multicolumn{4}{|c|}{ Main group } & \multicolumn{2}{|c|}{ Recovery group } \\
\hline & & Control & 30 & 100 & 300 & Control & 300 \\
\hline \multicolumn{8}{|l|}{ Males } \\
\hline \multicolumn{2}{|c|}{$\mathrm{RBC}\left(\times 10^{6}\right.$ cells $\left./ \mu \mathrm{L}\right)$} & $6.89 \pm 0.57$ & $6.92 \pm 0.55$ & $6.96 \pm 0.11$ & $6.57 \pm 0.34$ & $6.82 \pm 0.14$ & $6.62 \pm 0.06$ \\
\hline \multicolumn{2}{|l|}{$\operatorname{HGB}(\mathrm{g} / \mathrm{dL})$} & $15.8 \pm 1.1$ & $15.6 \pm 0.9$ & $15.6 \pm 0.7$ & $15.3 \pm 0.9$ & $16.1 \pm 0.9$ & $15.6 \pm 0.4$ \\
\hline \multicolumn{2}{|l|}{$\mathrm{HCT}(\%)$} & $43.9 \pm 3.3$ & $42.7 \pm 1.8$ & $43.2 \pm 1.6$ & $41.8 \pm 2.1$ & $44.1 \pm 1.6$ & $42.6 \pm 1.0$ \\
\hline \multicolumn{2}{|l|}{ Reti $(\%)$} & $0.79 \pm 0.14$ & $0.92 \pm 0.25$ & $0.68 \pm 0.11$ & $0.80 \pm 0.36$ & $1.16 \pm 0.13$ & $0.77 \pm 0.28$ \\
\hline \multirow[t]{3}{*}{$\mathrm{RBC}$ index } & MCV (fL) & $63.8 \pm 1.3$ & $61.8 \pm 2.6$ & $62.1 \pm 1.4$ & $63.6 \pm 1.3$ & $64.7 \pm 0.9$ & $64.4 \pm 1.9$ \\
\hline & MCH (pg) & $22.9 \pm 0.5$ & $22.6 \pm 1.0$ & $22.5 \pm 0.6$ & $23.2 \pm 0.6$ & $23.5 \pm 0.8$ & $23.5 \pm 0.7$ \\
\hline & MCHC (g/dL) & $35.9 \pm 0.4$ & $36.5 \pm 0.8$ & $36.3 \pm 0.5$ & $36.4 \pm 0.3$ & $36.4 \pm 0.8$ & $23.5 \pm 0.7$ \\
\hline \multicolumn{2}{|c|}{$\operatorname{PLT}\left(\times 10^{3}\right.$ cells $\left./ \mu \mathrm{L}\right)$} & $244 \pm 35$ & $273 \pm 8$ & $274 \pm 51$ & $309 \pm 86$ & $215 \pm 27$ & $255 \pm 16$ \\
\hline \multicolumn{2}{|c|}{$\mathrm{WBC}\left(\times 10^{3}\right.$ cells $\left./ \mu \mathrm{L}\right)$} & $9.08 \pm 1.99$ & $10.19 \pm 0.60$ & $10.59 \pm 2.44$ & $11.51 \pm 1.01$ & $7.90 \pm 1.24$ & $10.83 \pm 0.71$ \\
\hline WBC & NEU & $53.1 \pm 6.8$ & $49.8 \pm 11.5$ & $53.5 \pm 10.0$ & $57.5 \pm 4.0$ & $50.4 \pm 5.3$ & $35.7 \pm 6.6$ \\
\hline differential & LYM & $34.6 \pm 5.5$ & $37.4 \pm 9.5$ & $32.3 \pm 6.5$ & $31.8 \pm 2.5$ & $35.7 \pm 6.6$ & $33.2 \pm 5.5$ \\
\hline \multirow[t]{3}{*}{ counting $(\%)$} & MONO & $5.9 \pm 1.4$ & $7.9 \pm 2.2$ & $7.2 \pm 3.0$ & $6.0 \pm 1.6$ & $5.4 \pm 0.7$ & $7.0 \pm 0.2$ \\
\hline & EOS & $5.6 \pm 2.5$ & $3.9 \pm 0.5$ & $6.0 \pm 1.2$ & $3.6 \pm 2.0$ & $7.6 \pm 1.8$ & $7.0 \pm 0.2$ \\
\hline & BASO & $0.6 \pm 0.1$ & $0.7 \pm 0.2$ & $0.7 \pm 0.2$ & $0.6 \pm 0.3$ & $0.7 \pm 0.1$ & $0.8 \pm 0.3$ \\
\hline \multicolumn{2}{|l|}{ PT (sec) } & $10.5 \pm 1.1$ & $9.8 \pm 0.4$ & $9.6 \pm 0.1$ & $9.7 \pm 0.4$ & $10.0 \pm 0.7$ & $9.7 \pm 0.1$ \\
\hline \multicolumn{2}{|l|}{ APTT (sec) } & $16.0 \pm 1.1$ & $15.4 \pm 1.2$ & $14.4 \pm 1.5$ & $15.0 \pm 0.4$ & $14.9 \pm 3.3$ & $15.3 \pm 0.5$ \\
\hline \multicolumn{8}{|l|}{ Females } \\
\hline \multicolumn{2}{|c|}{$\mathrm{RBC}\left(\times 10^{6}\right.$ cells $\left./ \mu \mathrm{L}\right)$} & $6.60 \pm 0.26$ & $6.96 \pm 0.62$ & $6.37 \pm 0.17$ & $6.39 \pm 0.59$ & $7.60 \pm 0.09$ & $7.23 \pm 0.08$ \\
\hline \multicolumn{2}{|c|}{$\operatorname{HGB}(g / d L)$} & $14.9 \pm 0.8$ & $15.8 \pm 1.5$ & $14.4 \pm 0.9$ & $15.0 \pm 1.4$ & $17.5 \pm 0.6$ & $17.0 \pm 1.0$ \\
\hline \multicolumn{2}{|l|}{$\mathrm{HCT}(\%)$} & $41.4 \pm 1.6$ & $43.3 \pm 3.3$ & $40.0 \pm 2.4$ & $41.4 \pm 3.5$ & $47.6 \pm 0.8$ & $45.6 \pm 2.0$ \\
\hline \multicolumn{2}{|l|}{ Reti (\%) } & $0.64 \pm 0.19$ & $0.61 \pm 0.11$ & $0.83 \pm 0.20$ & $0.65 \pm 0.25$ & $0.83 \pm 0.19$ & $1.13 \pm 0.16$ \\
\hline \multirow{3}{*}{$\mathrm{RBC}$ index } & MCV (fL) & $62.7 \pm 1.6$ & $62.3 \pm 1.0$ & $62.7 \pm 2.3$ & $64.9 \pm 2.9$ & $62.7 \pm 1.8$ & $63.1 \pm 2.1$ \\
\hline & $\mathrm{MCH}(\mathrm{pg})$ & $22.6 \pm 0.9$ & $22.7 \pm 0.5$ & $22.6 \pm 0.8$ & $23.5 \pm 1.2$ & $23.1 \pm 1.1$ & $23.6 \pm 1.2$ \\
\hline & $\mathrm{MCHC}(\mathrm{g} / \mathrm{dL})$ & $36.0 \pm 0.7$ & $36.4 \pm 0.7$ & $36.0 \pm 0.3$ & $36.1 \pm 0.4$ & $36.9 \pm 0.6$ & $37.3 \pm 0.6$ \\
\hline \multicolumn{2}{|c|}{$\operatorname{PLT}\left(\times 10^{3}\right.$ cells $\left./ \mu \mathrm{L}\right)$} & $257 \pm 52$ & $338 \pm 42 *$ & $280 \pm 14$ & $307 \pm 25$ & $224 \pm 124$ & $302 \pm 21$ \\
\hline \multicolumn{2}{|c|}{$\mathrm{WBC}\left(\times 10^{3}\right.$ cells $\left./ \mu \mathrm{L}\right)$} & $8.75 \pm 2.01$ & $9.26 \pm 1.23$ & $10.68 \pm 2.21$ & $8.85 \pm 1.73$ & $6.95 \pm 0.73$ & $8.94 \pm 0.16$ \\
\hline WBC & NEU & $52.2 \pm 5.8$ & $57.0 \pm 10.2$ & $60.6 \pm 0.3$ & $56.5 \pm 10.0$ & $40.9 \pm 1.8$ & $50.4 \pm 4.9$ \\
\hline differential & LYM & $37.0 \pm 7.8$ & $33.6 \pm 9.5$ & $29.7 \pm 0.8$ & $33.7 \pm 9.7$ & $47.7 \pm 6.4$ & $37.4 \pm 7.6$ \\
\hline \multirow[t]{3}{*}{ counting $(\%)$} & MONO & $5.2 \pm 0.7$ & $5.1 \pm 1.4$ & $5.8 \pm 1.9$ & $5.8 \pm 3.0$ & $6.2 \pm 2.8$ & $4.3 \pm 0.2$ \\
\hline & EOS & $4.8 \pm 3.3$ & $5.1 \pm 1.4$ & $5.8 \pm 1.9$ & $5.8 \pm 0.7$ & $3.8 \pm 2.6$ & $6.8 \pm 2.6$ \\
\hline & BASO & $0.5 \pm 0.4$ & $0.9 \pm 0.6$ & $0.4 \pm 0.1$ & $0.6 \pm 0.2$ & $1.1 \pm 0.5$ & $0.9 \pm 0.1$ \\
\hline \multicolumn{2}{|l|}{ PT (sec) } & $9.7 \pm 0.1$ & $9.7 \pm 0.2$ & $11.2 \pm 1.8$ & $9.6 \pm 0.0$ & $9.9 \pm 0.2$ & $9.6 \pm 0.0$ \\
\hline \multicolumn{2}{|l|}{ APTT (sec) } & $17.4 \pm 1.7$ & $15.5 \pm 0.3$ & $16.4 \pm 1.3$ & $15.6 \pm 1.2$ & $16.1 \pm 0.3$ & $14.2 \pm 1.5$ \\
\hline
\end{tabular}

Significantly different from the control by Dunnett's t-test, $*: p<0.05$. 
No significant changes in urinalysis parameters were observed following LEH treatment in main and recovery group (Table 2 and 3 ).

In Week 4, PLT numbers were higher in females receiving $30 \mathrm{mg} \mathrm{LEH} / \mathrm{kg} /$ day, as compared to controls $(p<0.05)$ (Table 5). In males dosed with $30 \mathrm{mg} / \mathrm{kg} / \mathrm{day}, \alpha 1$-globulin and $\mathrm{P}$ were increased, while $\beta$-globulin was decreased $(p<$
$0.05)$. In males receiving $300 \mathrm{mg} / \mathrm{kg} /$ day, $\mathrm{P}$ was decreased $(p<0.05)$. T-Bili was also increased in males and females receiving 30 and $300 \mathrm{mg} / \mathrm{kg} /$ day $(p<0.05$ ) (qj 7). TG was increased in both sexes receiving $300 \mathrm{mg} / \mathrm{kg} /$ day $(p<0.01)$. In the recovery group, there were no abnormal changes in hematologic and blood biochemical parameters. The increased PLT in dogs receiving $30 \mathrm{mg} / \mathrm{kg}$ /day and the decreased $\mathrm{P}$

Table 6. Blood chemical values of Beagle dogs at week 0 (pre-dosing)

\begin{tabular}{|c|c|c|c|c|}
\hline Dose (mg/kg/day) & Control & 30 & 100 & 300 \\
\hline \multicolumn{5}{|l|}{ Males } \\
\hline $\operatorname{ALT}(\mathrm{U} / \mathrm{L})$ & $26.9 \pm 4.5$ & $28.4 \pm 1.1$ & $24.7 \pm 5.8$ & $25.7 \pm 6.7$ \\
\hline AST (U/L) & $28.7 \pm 2.6$ & $31.5 \pm 3.0$ & $26.1 \pm 3.4$ & $29.7 \pm 8.6$ \\
\hline ALP (U/L) & $465.6 \pm 90.4$ & $538.6 \pm 98.2$ & $490.1 \pm 92.9$ & $690.5 \pm 229.7$ \\
\hline GGT (U/L) & $3.65 \pm 0.28$ & $3.73 \pm 0.51$ & $3.79 \pm 0.71$ & $3.82 \pm 0.46$ \\
\hline Glu (mg/dL) & $107 \pm 5$ & $110 \pm 6.3$ & $103 \pm 4$ & $116 \pm 7$ \\
\hline BUN (mg/dL) & $9.7 \pm 1.0$ & $9.2 \pm 0.6$ & $10.8 \pm 0.3$ & $8.8 \pm 1.2$ \\
\hline Crea (mg/dL) & $0.69 \pm 0.03$ & $0.71 \pm 0.05$ & $0.73 \pm 0.07$ & $0.67 \pm 0.04$ \\
\hline T-Bili (mg/dL) & $0.02 \pm 0.01$ & $0.04 \pm 0.00$ & $0.01 \pm 0.01$ & $0.03 \pm 0.01$ \\
\hline T-Chol (mg/dL) & $171 \pm 22$ & $131 \pm 19$ & $166 \pm 52$ & $150 \pm 20$ \\
\hline TG (mg/dL) & $27 \pm 6$ & $26 \pm 3$ & $30 \pm 5$ & $28 \pm 3$ \\
\hline $\mathrm{TP}(\mathrm{mg} / \mathrm{dL})$ & $5.1 \pm 0.2$ & $5.0 \pm 0.2$ & $5.0 \pm 0.2$ & $5.1 \pm 0.2$ \\
\hline Alb (\%) & $45.5 \pm 4.0$ & $49.2 \pm 0.2$ & $45.7 \pm 2.8$ & $49.9 \pm 1.7$ \\
\hline Globulin- $\alpha 1(\%)$ & $11.4 \pm 1.0$ & $10.3 \pm 1.1$ & $10.2 \pm 0.9$ & $10.2 \pm 0.7$ \\
\hline Globulin- $\alpha 2(\%)$ & $13.1 \pm 1.1$ & $11.4 \pm 0.6$ & $13.2 \pm 1.7$ & $12.0 \pm 1.0$ \\
\hline Globulin- $\beta$ (\%) & $19.2 \pm 1.7$ & $19.2 \pm 0.3$ & $19.2 \pm 0.2$ & $17.6 \pm 1.3$ \\
\hline Globulin- $\gamma(\%)$ & $10.8 \pm 1.7$ & $9.8 \pm 0.4$ & $11.8 \pm 0.6$ & $10.3 \pm 1.8$ \\
\hline $\mathrm{A} / \mathrm{G}$ ration & $0.84 \pm 0.14$ & $0.97 \pm 0.01$ & $0.84 \pm 0.10$ & $1.00 \pm 0.07$ \\
\hline $\mathrm{P}(\mathrm{mg} / \mathrm{dL})$ & $6.55 \pm 0.54$ & $6.19 \pm 0.31$ & $6.52 \pm 0.46$ & $5.94 \pm 0.63$ \\
\hline $\mathrm{Ca}(\mathrm{mg} / \mathrm{dL})$ & $11.0 \pm 0.4$ & $11.1 \pm 0.2$ & $11.1 \pm 0.2$ & $11.0 \pm 0.2$ \\
\hline $\mathrm{Na}(\mathrm{mg} / \mathrm{dL})$ & $144.7 \pm 0.6$ & $143.9 \pm 0.3$ & $144.3 \pm 0.5$ & $144.1 \pm 0.9$ \\
\hline $\mathrm{K}(\mathrm{mg} / \mathrm{dL})$ & $4.59 \pm 0.23$ & $4.66 \pm 0.18$ & $4.94 \pm 0.14$ & $4.77 \pm 0.17$ \\
\hline $\mathrm{Cl}(\mathrm{mg} / \mathrm{dL})$ & $112.2 \pm 1.7$ & $110.1 \pm 0.9$ & $111.9 \pm 0.3$ & $110.1 \pm 0.3$ \\
\hline \multicolumn{5}{|l|}{ Females } \\
\hline ALT (U/L) & $26.6 \pm 2.1$ & $23.5 \pm 5.2$ & $24.6 \pm 0.8$ & $27.2 \pm 5.2$ \\
\hline AST (U/L) & $29.2 \pm 2.4$ & $28.5 \pm 6.0$ & $29.3 \pm 2.6$ & $34.8 \pm 8.2$ \\
\hline ALP (U/L) & $494.9 \pm 166.1$ & $360.7 \pm 76.9$ & $423.6 \pm 94.3$ & $443.5 \pm 161.1$ \\
\hline GGT (U/L) & $3.40 \pm 0.34$ & $3.55 \pm 0.52$ & $3.42 \pm 0.45$ & $3.70 \pm 0.97$ \\
\hline Glu (mg/dL) & $108 \pm 7$ & $110 \pm 4$ & $113 \pm 12$ & $106 \pm 6$ \\
\hline BUN (mg/dL) & $10.1 \pm 1.5$ & $11.3 \pm 1.9$ & $9.0 \pm 1.0$ & $10.7 \pm 0.9$ \\
\hline Crea (mg/dL) & $0.71 \pm 0.08$ & $0.73 \pm 0.03$ & $0.71 \pm 0.06$ & $0.72 \pm 0.08$ \\
\hline T-Bili (mg/dL) & $0.04 \pm 0.01$ & $0.03 \pm 0.01$ & $0.03 \pm 0.01$ & $0.04 \pm 0.02$ \\
\hline T-Chol (mg/dL) & $131 \pm 26$ & $139 \pm 27$ & $154 \pm 41$ & $130 \pm 12$ \\
\hline $\mathrm{TG}(\mathrm{mg} / \mathrm{dL})$ & $27 \pm 11$ & $25 \pm 5$ & $23 \pm 3$ & $24 \pm 7$ \\
\hline $\mathrm{TP}$ (mg/dL) & $5.0 \pm 0.2$ & $5.0 \pm 0.5$ & $5.1 \pm 0.0$ & $5.0 \pm 0.4$ \\
\hline Alb (\%) & $49.4 \pm 2.6$ & $51.8 \pm 0.7$ & $49.2 \pm 3.2$ & $49.8 \pm 1.4$ \\
\hline Globulin- $\alpha 1(\%)$ & $10.0 \pm 1.2$ & $10.5 \pm 0.8$ & $10.8 \pm 1.6$ & $8.5 \pm 1.7$ \\
\hline Globulin- $\alpha 2(\%)$ & $11.6 \pm 1.2$ & $11.5 \pm 0.6$ & $11.7 \pm 1.2$ & $13.0 \pm 1.5$ \\
\hline Globulin- $\beta$ (\%) & $18.6 \pm 1.2$ & $17.6 \pm 0.7$ & $18.8 \pm 0.9$ & $18.6 \pm 0.5$ \\
\hline Globulin- $\gamma(\%)$ & $10.5 \pm 1.8$ & $8.6 \pm 0.3$ & $9.5 \pm 1.8$ & $10.1 \pm 1.0$ \\
\hline $\mathrm{A} / \mathrm{G}$ ration & $0.98 \pm 0.10$ & $1.07 \pm 0.03$ & $0.97 \pm 0.13$ & $0.99 \pm 0.06$ \\
\hline $\mathrm{P}(\mathrm{mg} / \mathrm{dL})$ & $6.51 \pm 0.30$ & $6.62 \pm 0.81$ & $6.28 \pm 0.21$ & $6.05 \pm 0.62$ \\
\hline $\mathrm{Ca}(\mathrm{mg} / \mathrm{dL})$ & $10.6 \pm 0.1$ & $11.0 \pm 0.3$ & $10.9 \pm 0.2$ & $10.7 \pm 0.1$ \\
\hline $\mathrm{Na}(\mathrm{mg} / \mathrm{dL})$ & $143.4 \pm 1.1$ & $144.4 \pm 0.5$ & $144.2 \pm 0.7$ & $145.0 \pm 1.5$ \\
\hline $\mathrm{K}(\mathrm{mg} / \mathrm{dL})$ & $4.58 \pm 0.35$ & $4.82 \pm 0.40$ & $4.41 \pm 0.23$ & $4.51 \pm 0.36$ \\
\hline $\mathrm{Cl}(\mathrm{mg} / \mathrm{dL})$ & $109.8 \pm 1.9$ & $110.4 \pm 1.2$ & $111.2 \pm 2.1$ & $110.8 \pm 1.1$ \\
\hline
\end{tabular}


and $\beta$-globulin levels at 30 or $300 \mathrm{mg} / \mathrm{kg} / \mathrm{day}$, were not considered to be adverse effects; they were judged to be normal biological variations because these changes were within the ranges observed in historical background data (Biotox- tech background data, 2013) and were not accompanied by related histopathological changes. Increases in T-Bili and TG were considered to be of no toxicological significance, as these changes were observed during the dosing period

Table 7. Blood chemical values of Beagle dogs administered with Lithospermum erythrorhizon hexane extract or its vehicle

\begin{tabular}{|c|c|c|c|c|c|c|}
\hline \multirow{2}{*}{ Dose (mg/kg/day) } & \multicolumn{4}{|c|}{ Main group } & \multicolumn{2}{|c|}{ Recovery group } \\
\hline & Control & 30 & 100 & 300 & Control & 300 \\
\hline \multicolumn{7}{|l|}{ Males } \\
\hline ALT (U/L) & $28.8 \pm 2.4$ & $22.1 \pm 0.9$ & $210.0 \pm 5.0$ & $24.3 \pm 5.7$ & $30.4 \pm 4.7$ & $23.6 \pm 4.1$ \\
\hline AST (U/L) & $30.7 \pm 2.1$ & $29.8 \pm 3.0$ & $24.3 \pm 4.6$ & $29.1 \pm 7.0$ & $31.5 \pm 0.4$ & $28.0 \pm 2.1$ \\
\hline $\operatorname{ALP}(\mathrm{U} / \mathrm{L})$ & $388.9 \pm 89.9$ & $425.6 \pm 74.4$ & $430.1 \pm 77.5$ & $567.3 \pm 200.0$ & $404.0 \pm 169.4$ & $575.3 \pm 108.8$ \\
\hline GGT (U/L) & $4.13 \pm 0.28$ & $3.99 \pm 0.28$ & $4.37 \pm 0.51$ & $4.26 \pm 0.48$ & $4.02 \pm 0.34$ & $3.71 \pm 0.25$ \\
\hline Glu (mg/dL) & $102 \pm 4$ & $102 \pm 7$ & $105 \pm 7$ & $108 \pm 9.0$ & $98 \pm 1$ & $116 \pm 6$ \\
\hline BUN (mg/dL) & $10.3 \pm 1.6$ & $11.3 \pm 1.9$ & $12.5 \pm 2.4$ & $9.0 \pm 0.7$ & $10.6 \pm 1.3$ & $9.5 \pm 0.6$ \\
\hline Crea (mg/dL) & $0.74 \pm 0.03$ & $0.76 \pm 0.12$ & $0.76 \pm 0.12$ & $0.65 \pm 0.05$ & $0.76 \pm 0.02$ & $0.69 \pm 0.06$ \\
\hline T-Bili (mg/dL) & $0.03 \pm 0.00$ & $0.10 \pm 0.03^{\#}$ & $0.07 \pm 0.03$ & $0.15 \pm 0.07^{\#}$ & $0.02 \pm 0.01$ & $0.05 \pm 0.03$ \\
\hline T-Chol (mg/dL) & $170 \pm 6$ & $107 \pm 3$ & $176 \pm 74$ & $150 \pm 23$ & $161 \pm 1$ & $161 \pm 1$ \\
\hline $\mathrm{TG}(\mathrm{mg} / \mathrm{dL})$ & $28 \pm 8$ & $29 \pm 3$ & $45 \pm 4$ & $51 \pm 13^{* *}$ & $24 \pm 2$ & $24 \pm 2$ \\
\hline $\mathrm{TP}(\mathrm{mg} / \mathrm{dL})$ & $5.2 \pm 0.2$ & $5.2 \pm 0.2$ & $5.3 \pm 0.2$ & $5.1 \pm 0.1$ & $5.2 \pm 0.1$ & $5.1 \pm 0.2$ \\
\hline $\mathrm{Alb}(\%)$ & $47.4 \pm 2.2$ & $47.7 \pm 2.9$ & $45.9 \pm 1.7$ & $47.0 \pm 2.2$ & $45.0 \pm 3.2$ & $48.3 \pm 1.6$ \\
\hline Globulin- $\alpha 1(\%)$ & $11.1 \pm 0.5$ & $9.1 \pm 0.5^{*}$ & $11.1 \pm 1.5$ & $10.7 \pm 0.6$ & $11.6 \pm 0.7$ & $11.9 . \pm 0.0$ \\
\hline Globulin- $\alpha 2(\%)$ & $12.6 \pm 0.6$ & $12.3 \pm 0.7$ & $13.3 \pm 0.8$ & $12.7 \pm 0.7$ & $12.4 \pm 1.3$ & $11.9 \pm 0.1$ \\
\hline Globulin- $\beta(\%)$ & $18.6 \pm 0.8$ & $21.0 \pm 1.7^{*}$ & $19.7 \pm 0.4$ & $19.8 \pm 1.0$ & $20.1 \pm 2.0$ & $18.7 \pm 0.6$ \\
\hline Globulin- $\gamma(\%)$ & $10.4 \pm 2.0$ & $9.8 \pm 1.0$ & $10.0 \pm 0.3$ & $9.8 \pm 0.7$ & $11.0 \pm 0.6$ & $9.3 \pm 0.8$ \\
\hline $\mathrm{A} / \mathrm{G}$ ration & $0.90 \pm 0.08$ & $0.92 \pm 0.11$ & $0.85 \pm 0.06$ & $0.89 \pm 0.07$ & $0.82 \pm 0.10$ & $0.94 \pm 0.06$ \\
\hline $\mathrm{P}(\mathrm{mg} / \mathrm{dL})$ & $6.41 \pm 0.42$ & $5.48 \pm 0.72 *$ & $6.02 \pm 0.23$ & $5.67 \pm 0.25^{*}$ & $6.62 \pm 0.60$ & $6.29 \pm 0.01$ \\
\hline $\mathrm{Ca}(\mathrm{mg} / \mathrm{dL})$ & $10.8 \pm 0.4$ & $10.9 \pm 0.2$ & $11.2 \pm 0.2$ & $10.6 \pm 0.1$ & $10.8 \pm 0.4$ & $10.5 \pm 0.2$ \\
\hline $\mathrm{Na}(\mathrm{mg} / \mathrm{dL})$ & $144.2 \pm 1.0$ & $143.7 \pm 0.72$ & $143.7 \pm 1.0$ & $143.5 \pm 0.9$ & $144.3 \pm 0.7$ & $144.2 \pm 0.6$ \\
\hline $\mathrm{K}(\mathrm{mg} / \mathrm{dL})$ & $4.75 \pm 0.26$ & $4.74 \pm 0.20$ & $4.68 \pm 0.20$ & $4.85 \pm 0.42$ & $4.87 \pm 0.06$ & $4.77 \pm 0.08$ \\
\hline $\mathrm{Cl}(\mathrm{mg} / \mathrm{dL})$ & $111.9 \pm 1.9$ & $111.7 \pm 0.9$ & $111.3 \pm 0.7$ & $111.6 \pm 1.6$ & $115.0 \pm 0.6$ & $112.3 \pm 0.4$ \\
\hline \multicolumn{7}{|l|}{ Females } \\
\hline ALT (U/L) & $29.1 \pm 1.1$ & $20.7 \pm 6.9$ & $24.9 \pm 6.0$ & $23.1 \pm 2.6^{\#}$ & $29.7 \pm 2.1$ & $28.7 \pm 0.4$ \\
\hline AST (U/L) & $27.9 \pm 2.1$ & $23.2 \pm 3.8$ & $26.1 \pm 3.1$ & $28.5 \pm 4.8$ & $29.6 \pm 0.6$ & $37.8 \pm 6.0$ \\
\hline ALP (U/L) & $388.4 \pm 124.2$ & $290.2 \pm 77.0$ & $341.1 \pm 91.4$ & $330.0 \pm 100.4$ & $417.9 \pm 89.4$ & $421.8 \pm 246.6$ \\
\hline GGT (U/L) & $3.68 \pm 0.27$ & $3.69 \pm 0.52$ & $3.55 \pm 0.51$ & $3.88 \pm 0.56$ & $3.53 \pm 0.15$ & $5.12 \pm 1.34$ \\
\hline Glu (mg/dL) & $104 \pm 9$ & $99 \pm 3$ & $108 \pm 6$ & $96 \pm 9$ & $111 \pm 6$ & $100 \pm 2$ \\
\hline BUN (mg/dL) & $10.0 \pm 1.3$ & $10.2 \pm 0.9$ & $9.1 \pm 1.6$ & $9.7 \pm 0.7$ & $12.1 \pm 2.3$ & $11.6 \pm 1.3$ \\
\hline Crea (mg/dL) & $0.75 \pm 0.07$ & $0.72 \pm 0.05$ & $0.69 \pm 0.10$ & $0.69 \pm 0.08$ & $0.83 \pm 0.06$ & $0.77 \pm 0.11$ \\
\hline T-Bili (mg/dL) & $0.05 \pm 0.03$ & $0.09 \pm 0.03$ & $0.14 \pm 0.05$ & $0.27 \pm 0.14^{\#}$ & $0.05 \pm 0.01$ & $0.04 \pm 0.01$ \\
\hline T-Chol (mg/dL) & $129 \pm 21$ & $131 \pm 24$ & $141 \pm 35$ & $124 \pm 21$ & $127 \pm 7$ & $163 \pm 40$ \\
\hline $\mathrm{TG}(\mathrm{mg} / \mathrm{dL})$ & $25 \pm 6$ & $31 \pm 5$ & $36 \pm 13$ & $47 \pm 11^{* *}$ & $21 \pm 3$ & $39 \pm 4$ \\
\hline $\mathrm{TP}(\mathrm{mg} / \mathrm{dL})$ & $4.9 \pm 0.2$ & $4.9 \pm 0.4$ & $5.2 \pm 0.2$ & $5.0 \pm 0.3$ & $5.1 \pm 0.1$ & $5.4 \pm 0.1$ \\
\hline $\mathrm{Alb}(\%)$ & $50.9 \pm 2.4$ & $52.0 \pm 2.0$ & $49.2 \pm 3.4$ & $51.2 \pm 1.2$ & $52.2 \pm 1.3$ & $48.8 \pm 0.1$ \\
\hline Globulin- $\alpha 1(\%)$ & $10.6 \pm 1.0$ & $10.7 \pm 0.6$ & $10.9 \pm 1.5$ & $9.6 \pm 0.5$ & $10.4 \pm 0.9$ & $11.7 \pm 0.8$ \\
\hline Globulin- $\alpha 2(\%)$ & $10.6 \pm 1.0$ & $12.0 \pm 2.0$ & $11.2 \pm 1.0$ & $11.3 \pm 0.2$ & $10.5 \pm 0.6$ & $11.8 \pm 0.8$ \\
\hline Globulin- $\beta(\%)$ & $17.4 \pm 1.4$ & $17.4 \pm 0.9$ & $19.7 \pm 2.3$ & $18.5 \pm 0.7$ & $17.6 \pm 0.1$ & $18.8 \pm 0.3$ \\
\hline Globulin- $\gamma(\%)$ & $10.5 \pm 1.7$ & $9.0 \pm 1.6$ & $9.0 \pm 1.6$ & $9.4 \pm 0.6$ & $9.4 \pm 0.1$ & $9.0 \pm 1.4$ \\
\hline $\mathrm{A} / \mathrm{G}$ ration & $1.04 \pm 0.10$ & $1.09 \pm 0.09$ & $0.98 \pm 0.14$ & $1.05 \pm 0.05$ & $1.09 \pm 0.06$ & $0.96 \pm 0.06$ \\
\hline$P(\mathrm{mg} / \mathrm{dL})$ & $5.77 \pm 0.51$ & $6.52 \pm 0.37$ & $5.92 \pm 0.07$ & $5.50 \pm 0.67$ & $6.64 \pm 0.34$ & $5.97 \pm 1.34$ \\
\hline $\mathrm{Ca}(\mathrm{mg} / \mathrm{dL})$ & $10.4 \pm 0.2$ & $11.0 \pm 0.1^{* *}$ & $10.7 \pm 0.3$ & $10.3 \pm 0.2$ & $10.8 \pm 0.1$ & $10.8 \pm 0.7$ \\
\hline $\mathrm{Na}(\mathrm{mg} / \mathrm{dL})$ & $143.0 \pm 1.9$ & $144.5 \pm 0.4$ & $144.6 \pm 0.8$ & $144.0 \pm 2.0$ & $142.2 \pm 1.4$ & $145.3 \pm 1.6$ \\
\hline $\mathrm{K}(\mathrm{mg} / \mathrm{dL})$ & $4.63 \pm 0.41$ & $4.89 \pm 0.25$ & $4.45 \pm 0.04$ & $4.56 \pm 0.41$ & $5.01 \pm 0.45$ & $4.69 \pm 0.01$ \\
\hline $\mathrm{Cl}(\mathrm{mg} / \mathrm{dL})$ & $110.8 \pm 2.4$ & $111.3 \pm 1.1$ & $110.9 \pm 1.7$ & $112.0 \pm 2.6$ & $111.0 \pm 0.2$ & $111.9 \pm 3.1$ \\
\hline
\end{tabular}

Significantly different from the control by Dunnett's t-test : ${ }^{*} p<0.05,{ }^{* *} p<0.01$.

Significantly different from the control by Steel's test: ${ }^{*} p<0.05$. 
Table 8. Absolute and relative organ weight in male Beagle dogs

\begin{tabular}{|c|c|c|c|c|c|c|}
\hline \multirow{2}{*}{ Dose (mg/kg/day) } & \multicolumn{4}{|c|}{ Main group } & \multicolumn{2}{|c|}{ Recovery group } \\
\hline & Control & 30 & 100 & 300 & Control & 300 \\
\hline Brain (g) & $82.0 \pm 8.2$ & $84.2 \pm 4.1$ & $72.9 \pm 4.5$ & $73.6 \pm 3.0$ & $79.9 \pm 1.5$ & $72.4 \pm 0.6$ \\
\hline Pituitary (mg) & $63.1 \pm 4.3$ & $68.9 \pm 12.9$ & $73.6 \pm 14.3$ & $69.1 \pm 9.8$ & $68.5 \pm 2.3$ & $68.5 \pm 2.3$ \\
\hline Thyroid (g) & $0.754 \pm 0.197$ & $0.833 \pm 0.359$ & $0.745 \pm 0.225$ & $0.805 \pm 0.103$ & $0.822 \pm 0.128$ & $0.996 \pm 0.277$ \\
\hline Thymus (g) & $6.81 \pm 2.51$ & $4.50 \pm 1.31$ & $6.96 \pm 2.72$ & $6.25 \pm 2.54$ & $10.81 \pm 1.96$ & $10.73 \pm 1.03$ \\
\hline Heart $(\mathrm{g})$ & $62.5 \pm 4.8$ & $64.1 \pm 4.8$ & $64.4 \pm 11.2$ & $55.9 \pm 3.6$ & $64.3 \pm 4.3$ & $59.7 \pm 7.4$ \\
\hline Liver (g) & $230 \pm 21$ & $233 \pm 3$ & $267 \pm 40$ & $239 \pm 33$ & $231 \pm 4$ & $240 \pm 25$ \\
\hline Spleen $(\mathrm{g})$ & $241 \pm 24.1$ & $24.8 \pm 3.8$ & $25.5 \pm 7.4$ & $31.1 \pm 10.8$ & $30.4 \pm 1.5$ & $23.7 \pm 1.0$ \\
\hline Kidney (g) & $41.0 \pm 1.8$ & $39.9 \pm 3.6$ & $39.3 \pm 3.4$ & $41.9 \pm 3.9$ & $40.6 \pm 2.9$ & $41.2 \pm 0.6$ \\
\hline Adrenal (g) & $1.185 \pm 0.222$ & $1.219 \pm 0.341$ & $0.992 \pm 0.082$ & $1.057 \pm 0.279$ & $1.493 \pm 0.025$ & $0.897 \pm 0.030$ \\
\hline Testis (g) & $8.02 \pm 1.52$ & $5.99 \pm 1.08$ & $8.17 \pm 0.87$ & $8.50 \pm 2.92$ & $9.66 \pm 0.25$ & $10.89 \pm 2.16$ \\
\hline Epididymis (g) & $1.69 \pm 0.27$ & $1.29 \pm 0.03$ & $2.02 \pm 0.27$ & $1.76 \pm 0.26$ & $2.06 \pm 0.37$ & $1.57 \pm 0.62$ \\
\hline Prostate (g) & $2.86 \pm 0.34$ & $1.37 \pm 0.25$ & $4.01 \pm 1.52$ & $2.39 \pm 0.91$ & $3.27 \pm 1.30$ & $3.22 \pm 2.05$ \\
\hline \multirow{2}{*}{ Dose (mg/kg/day) } & \multicolumn{4}{|c|}{ Main group } & \multicolumn{2}{|c|}{ "Recovery group } \\
\hline & Control & 30 & 100 & 300 & Control & 300 \\
\hline Brain $(\%)$ & $0.89 \pm 0.12$ & $0.97 \pm 0.07$ & $0.82 \pm 0.09$ & $0.86 \pm 0.52$ & $0.86 \pm 0.03$ & $0.82 \pm 0.04$ \\
\hline Pituitary (\%o) & $0.0069 \pm 0.0003$ & $0.0079 \pm 0.0014$ & $0.0082 \pm 0.0018$ & $0.0081 \pm 0.014$ & $0.0073 \pm 0.0001$ & $0.0075 \pm 0.0000$ \\
\hline Thyroid (\%o) & $0.082 \pm 0.021$ & $0.097 \pm 0.044$ & $0.082 \pm 0.019$ & $0.094 \pm 0.015$ & $0.089 \pm 0.018$ & $0.113 \pm 0.026$ \\
\hline Thymus (\%o) & $0.747 \pm 0.287$ & $0.522 \pm 0.166$ & $0.765 \pm 0.243$ & $0.720 \pm 0.255$ & $1.154 \pm 0.149$ & $1.223 \pm 0.170$ \\
\hline Heart (\%) & $0.68 \pm 0.03$ & $0.74 \pm 0.06$ & $0.71 \pm 0.09$ & $0.65 \pm 0.04$ & $0.69 \pm 0.01$ & $0.68 \pm 0.06$ \\
\hline Liver (\%) & $2.51 \pm 0.14$ & $2.70 \pm 0.03$ & $2.97 \pm 0.25$ & $2.80 \pm 0.46$ & $2.48 \pm 0.09$ & $2.72 \pm 0.16$ \\
\hline Spleen $(\%)$ & $0.26 \pm 0.05$ & $0.29 \pm 0.05$ & $0.28 \pm 0.06$ & $0.36 \pm 0.14$ & $0.33 \pm 0.01$ & $0.27 \pm 0.03$ \\
\hline Kidney (\%) & $0.45 \pm 0.01$ & $0.46 \pm 0.03$ & $0.43 \pm 0.01$ & $0.49 \pm 0.07$ & $0.44 \pm 0.01$ & $0.47 \pm 0.03$ \\
\hline Adrenal (\%o) & $0.129 \pm 0.023$ & $0.141 \pm 0.037$ & $0.111 \pm 0.005$ & $0.124 \pm 0.038$ & $0.160 \pm 0.006$ & $0.102 \pm 0.001$ \\
\hline Testis (\%o) & $0.874 \pm 0.142$ & $0.695 \pm 0.142$ & $0.912 \pm 0.101$ & $1.005 \pm 0.386$ & $1.037 \pm 0.082$ & $1.244 \pm 0.229$ \\
\hline Epididymis (\%o) & $0.185 \pm 0.024$ & $0.149 \pm 0.002$ & $0.225 \pm 0.021$ & $0.206 \pm 0.038$ & $0.220 \pm 0.29$ & $0.180 \pm 0.077$ \\
\hline Prostate $(\%)$ & $0.314 \pm 0.048$ & $0.159 \pm 0.029$ & $0.442 \pm 0.0138$ & $0.278 \pm 0.110$ & $0.347 \pm 0.122$ & $0.371 \pm 0.249$ \\
\hline
\end{tabular}

only and were not accompanied by histopathological changes in the liver.

The absolute weight of the uterus was significantly increased in females at $100 \mathrm{mg} / \mathrm{kg} / \mathrm{day}(p<0.05)$ (Table 9). The other absolute and relative (organ to body-weight ratio) weights of the organs were not significantly different between the control and treated animals. The increase in the absolute weight of the uterus at $100 \mathrm{mg} / \mathrm{kg} / \mathrm{day}$ was not considered to be meaningful because there was no significant change in the relative organ weight of the uterus and the effect was not dose-dependent.

In the LEH-treated groups, black spots, foci, and/or areas were observed in the lungs. These lesions were related to hemorrhage or inflammation, with alveolar phagocytosis of acidophilic material and/or brown pigmentation. These changes were not test-related adverse

Table 9. Absolute and relative organ weight in female Beagle dogs

\begin{tabular}{|c|c|c|c|c|c|c|}
\hline \multirow{2}{*}{ Dose (mg/kg/day) } & \multicolumn{4}{|c|}{ Main group } & \multicolumn{2}{|c|}{ Recovery group } \\
\hline & Control & 30 & 100 & 300 & Control & 300 \\
\hline Brain $(g)$ & $73.0 \pm 10.0$ & $73.5 \pm 4.6$ & $68.0 \pm 3.7$ & $75.2 \pm 5.8$ & $74.2 \pm 4.4$ & $75.9 \pm 6.6$ \\
\hline Pituitary (mg) & $59.5 \pm 1.3$ & $62.5 \pm 5.0$ & $64.5 \pm 3.5$ & $62.0 \pm 1.5$ & $61.4 \pm 1.6$ & $73.6 \pm 22.4$ \\
\hline Thyroid (g) & $0.731 \pm 0.185$ & $0.719 \pm 0.251$ & $0.779 \pm 0.204$ & $0.630 \pm 0.109$ & $0.886 \pm 0.190$ & $0.854 \pm 0.016$ \\
\hline Thymus (g) & $6.30 \pm 3.10$ & $7.71 \pm 6.26$ & $5.86 \pm 1.13$ & $4.45 \pm 1.97$ & $14.06 \pm 6.58$ & $5.67 \pm 0.43$ \\
\hline Heart $(\mathrm{g})$ & $55.2 \pm 58.3$ & $58.3 \pm 9.8$ & $58.1 \pm 8.4$ & $54.8 \pm 6.5$ & $62.7 \pm 3.0$ & $57.9 \pm 5.2$ \\
\hline Liver (g) & $182 \pm 31$ & $191 \pm 21$ & $216 \pm 35$ & $179 \pm 13$ & $187 \pm 0$ & $197 \pm 31$ \\
\hline Spleen $(\mathrm{g})$ & $24.4 \pm 0.6$ & $25.8 \pm 5.4$ & $25.3 \pm 2.9$ & $24.2 \pm 5.1$ & $28.1 \pm 1.3$ & $22.2 \pm 4.0$ \\
\hline Kidney (g) & $33.3 \pm 2.3$ & $34.0 \pm 2.4$ & $34.2 \pm 3.5$ & $34.1 \pm 3.5$ & $31.4 \pm 0.7$ & $36.1 \pm 2.7$ \\
\hline Adrenal (g) & $1.023 \pm 0.204$ & $1.103 \pm 0.058$ & $1.081 \pm 0.198$ & $1.096 \pm 0.090$ & $1.052 \pm 0.111$ & $1.142 \pm 0.030$ \\
\hline Ovary $(\mathrm{g})$ & $0.512 \pm 0.054$ & $0.511 \pm 0.042$ & $0.631 \pm 631^{*}$ & $0.491 \pm 0.038$ & $0.556 \pm 0.043$ & $0.497 \pm 0.021$ \\
\hline Uterus (g) & $2.29 \pm 1.05$ & $1.20 \pm 1.78$ & $1.78 \pm 1.0$ & $1.69 \pm 1.22$ & $2.31 \pm 0.60$ & $1.70 \pm 1.34$ \\
\hline
\end{tabular}


Table 9. Continued

\begin{tabular}{|c|c|c|c|c|c|c|}
\hline \multirow{2}{*}{ Dose (mg/kg/day) } & \multicolumn{4}{|c|}{ Main group } & \multicolumn{2}{|c|}{ Recovery group } \\
\hline & Control & 30 & 100 & 300 & Control & 300 \\
\hline Brain (\%) & $0.95 \pm 0.15$ & $0.96 \pm 0.03$ & $0.91 \pm 0.09$ & $1.07 \pm 0.17$ & $0.90 \pm 0.11$ & $0.99 \pm 0.04$ \\
\hline Pituitary (\%o) & $0.0078 \pm 0.0006$ & $0.0082 \pm 0.0007$ & $0.0086 \pm 0.0003$ & $0.0088 \pm 0.0011$ & $0.0074 \pm 0.0003$ & $0.0095 \pm 0.0016$ \\
\hline Thyroid (\%o) & $0.095 \pm 0.026$ & $0.095 \pm 0.036$ & $0.105 \pm 0.032$ & $0.088 \pm 0.007$ & $0.108 \pm 0.030$ & $0.112 \pm 0.017$ \\
\hline Thymus (\%o) & $0.808 \pm 0.349$ & $1.006 \pm 0.809$ & $0.786 \pm 0.165$ & $0.620 \pm 0.251$ & $1.675 \pm 0.688$ & $0.740 \pm 0.042$ \\
\hline Heart (\%) & $0.72 \pm 0.05$ & $0.76 \pm 0.11$ & $0.78 \pm 0.11$ & $0.77 \pm 0.02$ & $0.76 \pm 0.01$ & $0.76 \pm 0.04$ \\
\hline Liver (\%) & $2.39 \pm 0.53$ & $2.50 \pm 0.19$ & $2.90 \pm 0.55$ & $2.54 \pm 0.38$ & $2.26 \pm 0.14$ & $2.56 \pm 0.06$ \\
\hline Spleen $(\%)$ & $0.32 \pm 0.02$ & $0.34 \pm 0.06$ & $0.34 \pm 0.04$ & $0.34 \pm 0.07$ & $0.38 \pm 0.01$ & $0.29 \pm 0.01$ \\
\hline Kidney (\%) & $0.44 \pm 0.05$ & $0.45 \pm 0.02$ & $0.46 \pm 0.06$ & $0.48 \pm 0.02$ & $0.128 \pm 0.022$ & $0.47 \pm 0.03$ \\
\hline Adrenal (\%o) & $0.135 \pm 0.035$ & $0.145 \pm 0.007$ & $0.145 \pm 0.029$ & $0.156 \pm 0.026$ & $0.067 \pm 0.001$ & $0.150 \pm 0.016$ \\
\hline Ovary (\%o) & $0.067 \pm 0.009$ & $0.067 \pm 0.006$ & $0.084 \pm 0.009$ & $0.069 \pm 0.006$ & $0.067 \pm 0.001$ & $0.065 \pm 0.006$ \\
\hline Uterus (\%) & $0.304 \pm 0.153$ & $0.157 \pm 0.090$ & $0.235 \pm 0.135$ & $0.230 \pm 0.161$ & $0.276 \pm 0.055$ & $0.212 \pm 0.146$ \\
\hline
\end{tabular}

Significantly different from the control by Dunnett's t-test: ${ }^{*} p<0.05$.

Table 10. Toxicokinetic parameters for acetylshikonin in dog plasma on Day 1 and Day 28

\begin{tabular}{|c|c|c|c|c|c|c|}
\hline \multirow{2}{*}{ Sex } & \multirow{2}{*}{$\begin{array}{c}\text { Dose } \\
\text { (mg/kg/day) }\end{array}$} & \multirow{2}{*}{ Phase } & \multicolumn{4}{|c|}{ Toxicokinetic parameters } \\
\hline & & & $\mathrm{AUC}_{\text {last }}(\mathrm{ng} \cdot \mathrm{hr} / \mathrm{mL})$ & $\mathrm{C}_{\max }(\mathrm{ng} / \mathrm{mL})$ & $\mathrm{T}_{\max }(\mathrm{hr})$ & $\mathrm{t}_{1 / 2}(\mathrm{hr})$ \\
\hline \multirow[t]{6}{*}{ Males } & 30 & Day 1 & 98.06 & 31.33 & 0.67 & 2.49 \\
\hline & & Day 28 & 413.06 & 42.37 & 1.50 & 11.51 \\
\hline & 100 & Day 1 & 260.32 & 37.50 & 0.75 & 5.25 \\
\hline & & Day 28 & 1202.06 & 88.17 & 0.83 & 12.74 \\
\hline & 300 & Day 1 & 138.46 & 37.40 & 1.00 & 4.91 \\
\hline & & Day 28 & 1427.68 & 142.67 & 1.67 & 12.46 \\
\hline \multirow{6}{*}{ Females } & 30 & Day 1 & 502.42 & 60.70 & 3.33 & 6.90 \\
\hline & & Day 28 & 452.89 & 44.67 & 2.33 & 16.31 \\
\hline & 100 & Day 1 & 356.48 & 43.80 & 1.58 & 8.77 \\
\hline & & Day 28 & 1156.48 & 82.07 & 2.25 & 15.11 \\
\hline & 300 & Day 1 & 361.67 & 56.20 & 1.08 & 6.93 \\
\hline & & Day 28 & 1330.31 & 120.10 & 1.50 & 17.17 \\
\hline
\end{tabular}

effects because the acidophilic material appeared the test substance which induced vomiting and influx into the lung. A red focus was observed in one male treated with $30 \mathrm{mg} / \mathrm{kg} / \mathrm{day}$. This lesion arose from mucosal congestion and was considered to be incidental. The other lesions were considered to be spontaneous and of no toxicological significance.

Toxicokinetic studies (Table 10) found that blood concentrations of acetylshikonin, as an index analyte, were dose-dependently increased on Day 28, but there was no dose-dependent increase on Day 1. There were no gender differences; however, repeat dosing resulted in test substance accumulation on Day 28, as compared to Day 1.

In conclusion, the results indicated that sub-acute administration of LEH at a dose of $100 \mathrm{mg} / \mathrm{kg} /$ day or less did not produce any toxic effects in dogs. These data suggested that the no observed adverse effect level (NOAEL) of LEH was $100 \mathrm{mg} / \mathrm{kg} /$ day in Beagle dogs.

\section{ACKNOWLEDGMENT}

This research was supported by Bio-industry Technology Development Program, Ministry of Agriculture, Food and Rural Affairs (311054-3).

\section{REFERENCES}

1. Chen, X., Yang, L., Oppenheim, J.J. and Howard, M.Z. (2002) Cellular pharmacology studies of shikonin derivatives. Phytother. Res., 16, 199-209.

2. Rajasekar, S., Park D.J., Park, C., Park, S., Park, Y.H., Kim, S.T., Choi, Y.H. and Choi, Y.W. (2012) In vitro and in vivo anticancer effects of Lithospermum erythrorhizon extract on B16F10 murine melanoma. J. Ethnopharmacol., 144, 335345.

3. Lee, C.C., Wang, C.N., Lai, Y.T., Kang, J.J., Liao, J.W., Chiang, B.L., Chen, H.C. and Cheng, Y.W. (2010) Shikonin inhibits maturation of bone marrow-derived dendritic cells and suppresses allergic airway inflammation in a murine 
model of asthma. Br. J. Pharmacol., 161, 1496-1511.

4. Andújar, I., Ríos, J.L., Giner, R.M. and Recio, M.C. (2013) Shikonin promotes intestinal wound healing in vitro via induction of TGF- $\beta$ release in IEC-18 cells. Eur. J. Pharm. Sci., 49, 637-641.

5. Karayannopoulou, M., Tsioli, V., Loukopoulos, P., Anagnostou, T.L., Giannakas, N., Savvas, I., Papazoglou, L.G. and Kaldrymidou, E. (2011) Evaluation of the effectiveness of an ointment based on Alkannins/Shikonins on second intention wound healing in the dog. Can. J. Vet. Res., 75, 42-48.

6. Min, R., Tong, J., Wenjun, Y., Wenhu, D., Xiaojian, Z., Jiacai, H., Jian, Z., Wantao, C. and Chenping, Z. (2008) Growth inhibition and induction of apoptosis in human oral squamous cell carcinoma Tca- 8113 cell lines by Shikonin was partly through the inactivation of NF-kappaB pathway. Phytother. Res., 22, 407-415.

7. Yingkun, N., Lysong, Z. and Huimin, Y. (2010) Shikonin inhibits the proliferation and induces the apoptosis of human HepG2 cells. Can. J. Physiol. Pharmacol., 88, 1138-1146.

8. Chang, I.C., Huang, Y.J., Chiang, T.I., Yeh, C.W. and Hsu, L.S. (2010) Shikonin induces apoptosis through reactive oxy- gen species/extracellular signal-regulated kinase pathway in osteosarcoma cells. Biol. Pharm. Bull., 33, 816-824.

9. Mao, X., Yu, C.R., Li, W.H. and Li, W.X. (2008) Induction of apoptosis by shikonin through a ROS/JNK-mediated process in Bcr/Abl-positive chronic myelogenous leukemia (CML) cells. Cell Res., 18, 879-888.

10. Yang, H., Zhou, P., Huang, H., Chen, D., Ma, N., Cui, Q.C., Shen, S., Dong, W., Zhang, X., Lian, W., Wang, X., Dou, Q.P. and Liu, J. (2009) Shikonin exerts antitumor activity via proteasome inhibition and cell death induction in vitro and in vivo. Int. J. Cancer, 124, 2450-2459.

11. Lee, H.J., Lee, H.J., Magesh, V., Nam, D., Lee, E.O., Ahn, K.S., Jung, M.H., Ahn, K.S., Kim, D.K., Kim, J.Y. and Kim, S.H. (2008) Shikonin, acetylshikonin, and isobutyroylshikonin inhibit VEGF-induced angiogenesis and suppress tumor growth in lewis lung carcinoma-bearing mice. Yakugaku Zasshi, 128, 1681-1688.

12. Chen, C.H., Lin, M.L., Ong, P.L. and Yang, J.T. (2012) Novel multiple apoptotic mechanism of shikonin in human glioma cells. Ann. Surg. Oncol., 19, 3097-3106. 